\title{
Human Alzheimer's disease gene expression signatures and immune profile in APP mouse models: a discrete transcriptomic view of $A \beta$ plaque pathology
}

Sarah M. Rothman ${ }^{1}$, Keith Q. Tanis², Pallavi Gandhi ${ }^{3}$, Vladislav Malkov ${ }^{4}$, Jacob Marcus ${ }^{5}$, Michelle Pearson ${ }^{5}$, Richard Stevens ${ }^{4}$, Jason Gilliland ${ }^{2}$, Christopher Ware', Veeravan Mahadomrongkul ${ }^{3}$, Elaine O'Loughlin ${ }^{3}$, Gonzalo Zeballos ${ }^{3}$, Roger Smith ${ }^{6}$, Bonnie J. Howell ${ }^{7}$, Joel Klappenbach", Matthew Kennedy ${ }^{3}$ and Christian Mirescu ${ }^{3 *}$

\begin{abstract}
Background: Alzheimer's disease (AD) is a chronic neurodegenerative disease with pathological hallmarks including the formation of extracellular aggregates of amyloid-beta $(A \beta)$ known as plaques and intracellular tau tangles. Coincident with the formation of $A \beta$ plaques is recruitment and activation of glial cells to the plaque forming a plaque niche. In addition to histological data showing the formation of the niche, AD genetic studies have added to the growing appreciation of how dysfunctional glia pathways drive neuropathology, with emphasis on microglia pathways. Genomic approaches enable comparisons of human disease profiles between different mouse models informing on their utility to evaluate secondary changes to triggers such as A deposition.
\end{abstract}

Methods: In this study, we utilized two animal models of AD to examine and characterize the AD-associated pathology: the Tg2576 Swedish APP (KM670/671NL) and TgCRND8 Swedish plus Indiana APP (KM670/671NL + V717F) lines. We used laser capture microscopy (LCM) to isolate samples surrounding Thio-S positive plaques from distal non-plaque tissue. These samples were then analyzed using RNA sequencing.

Results: We determined age-associated transcriptomic differences between two similar yet distinct APP transgenic mouse models, known to differ in proportional amyloidogenic species and plaque deposition rates. In Tg2576, human AD gene signatures were not observed despite profiling mice out to 15 months of age. TgCRND8 mice however showed progressive and robust induction of lysomal, neuroimmune, and ITIM/ITAM-associated gene signatures overlapping with prior human AD brain transcriptomic studies. Notably, RNAseq analyses highlighted the vast majority of transcriptional changes observed in aging TgCRND8 cortical brain homogenates were in fact specifically enriched within the plaque niche samples. Data uncovered plaque-associated enrichment of microglia-related genes such as ITIM/TAMM-associated genes and pathway markers of phagocytosis.

Conclusion: This work may help guide improved translational value of APP mouse models of AD, particularly for strategies aimed at targeting neuroimmune and neurodegenerative pathways, by demonstrating that TgCRND8 more closely recapitulates specific human AD-associated transcriptional responses.

Keywords: Alzheimer's disease, Transcriptomics, Plaque, Microglia, Neuroinflammation

\footnotetext{
* Correspondence: christian_mirescu@merck.com

${ }^{3}$ Neuroscience, Merck \& Co., Merck Research Labs, Boston, Massachusetts,

USA

Full list of author information is available at the end of the article
}

(c) The Author(s). 2018 Open Access This article is distributed under the terms of the Creative Commons Attribution 4.0 International License (http://creativecommons.org/licenses/by/4.0/), which permits unrestricted use, distribution, and reproduction in any medium, provided you give appropriate credit to the original author(s) and the source, provide a link to the Creative Commons license, and indicate if changes were made. The Creative Commons Public Domain Dedication waiver (http://creativecommons.org/publicdomain/zero/1.0/) applies to the data made available in this article, unless otherwise stated. 


\section{Background}

Alzheimer's disease (AD) is a progressive, neurodegenerative disease characterized by a wide spectrum of pathological hallmarks including amyloid-beta $(\mathrm{A} \beta)$ plaques, intraneuronal neurofibrillary tangles, atrophy of synapses, and dendritic arbors, with associated cognitive decline [1]. While histological studies have characterized the morphology and process complexity of glial cells in a wide-range of neurodegenerative diseases, specific genomic and proteomic data regarding how microglia and astrocytes are modulated by disease, and vice versa, remain unknown. The lack of data regarding microglial reactivity in neurodegeneration is somewhat due to controversy regarding the utility of specific AD mouse models as windows into or prototype for human disease, and a paucity of studies using panoramic unbiased molecular profiling approaches to better describe local pathological changes occurring within the $A \beta$ niche.

Numerous studies using mouse AD models have identified and pre-clinically validated mechanisms targeted at either neuro-inflammatory or innate immune pathways [25]. However, the predictive value of these data is predicated on the translational relevance of the selected mouse model. Hence, clear understanding of how molecular pathways, particularly those related to the neuroimmune response, change with respect to disease pathology in these models is a key to accurate data analysis and interpretation. RNAseq is well suited to mapping out transcriptome changes across the entire genome and has significant advantages to low-throughout platforms and microarrays which are biased to known transcripts and generally remain limited to comparative inferences. One goal of the current study was to complete longitudinal evaluation of two different APP mouse models using RNAseq in brain regions demonstrating heavy plaque burden to evaluate their overlap with gene expression signatures previously identified in human $\mathrm{AD}$ brain samples.

Microglia are the brain's resident immune cells that maintain central nervous system (CNS) homeostasis, constantly survey their environment, and react to injury by initiating an inflammatory reaction [6]. They express a diverse set of pattern recognition receptors capable of sensing these damage-associated signals and pathogens in the extracellular milieu and respond to neuronal injury and tissue pathogens by rapidly extending processes toward and chemotaxing into the pathological niche. Not surprisingly, in the AD brain, microglia cluster and surround $A \beta$ plaques, a phenomenon also noted in many mouse models [7-9]. Despite the similar observations between mouse and humans, it remains to be seen how the plaque niche in $\mathrm{AD}$ mouse models may be dysregulated with regard to expression of human $\mathrm{AD}$ signatures, inflammatory versus innate immune pathways, and what transcriptome-wide profiling insights can be gained about biological changes occurring in the plaque-associated tissue. Notably, drugs targeting inflammation in $\mathrm{AD}$, such as $\mathrm{COX}$ inhibitors and nonsteroidal anti-inflammatory drugs (NSAIDs), have failed in the clinic underscoring the need for validating translational relevance of mouse models with respect to neuroinflammation such that novel insights [10-14], therapeutic hypotheses, and targets are more likely to translate to clinical success.

Large-scale AD GWAS studies have provided genetic hypotheses for neuroinflammation in AD [1523] and have consequently altered the perspective away from classical inflammatory pathways and toward innate immune dysfunction. Further support for a role of innate immune dysfunction in AD lies in recent transcriptomic characterization of $\mathrm{AD}$ brains which identified strong induction of an immune/microglia-related gene network in AD subjects [24]. Deeper characterization using directed probabilistic Bayesian networks identified the immune/microglia network as the module most strongly associated with the pathophysiology of Late-Onset Alzheimer's disease (LOAD). This Bayesian network consists of five immunologic signaling families including the family of genes associated with complement signaling which included TYROBP, TREM2, and CD33 [23]. Loss of function mutations, specifically in TREM2 and TYROBP, linked with neurodegeneration originally via Nasu-Hakola disease and frontotemporal dementia were subsequently confirmed by several GWAS studies [25-28]. TREM2 and CD33, both extracellular microglial-enriched receptors associated with innate immune signaling, are known drivers of human AD pathogenesis [16-18, 29]. Follow-up functional studies of TREM2 and CD33 biology provide converging data demonstrating that defects in A $\beta$ clearance may underlie the microglial-associated risk of AD pathophysiology (reviewed in [22]).

TREM2 and CD33 expressing microglia are known to localize around $\mathrm{A} \beta$ plaques in $\mathrm{AD}$ [30-32], and as such, we utilized laser capture microdissection to focus our current molecular profiling on the $A \beta$ pathological niche. We provide a contextual view of tissue gene expression in a mouse model of AD with confirmed overlap with human AD-associated signatures. We hypothesize that beyond the enrichment of causal human AD genes, there exists additional genetically associated biology relevant to the development of AD pathology. Accordingly, data regarding alterations in gene expression around areas of plaque deposition could inform on local networks for potential therapeutic intervention as well as improve our understanding of local $A \beta$-related changes to enable better model systems to validate hypotheses in future studies. 


\section{Methods \\ Animals}

Male transgenic (Tg) mice expressing APP KM670/671NL

(Swe) and APP V717F (TgCRND8) and age-matched wild-type (WT) littermates were used in whole cortex and laser capture RNAseq evaluations as well as in situ hybridization studies. Tg female mice carrying the APPSwe mutation (Tg2576) [33] and age-matched WT littermates were used for the whole-cortex evaluations. Mixed sex cohorts were not available, precluding any evaluation of sex differences in gene expression. Mice were bred for Merck at Taconic Farms (Hudson, NY, USA) and single housed in an Association for Assessment and Accreditation of Laboratory Animal Care accredited facility that was maintained on a 12-h light/dark cycle (lights on at $0700 \mathrm{~h}$ ). Food and water were available ad libitum. Principles of laboratory animal care were followed, and all studies were previously approved by the Institutional Animal Care and Use Committee and were performed in accordance to the Guide for the Care and Use of Laboratory Animals as adopted and promulgated by the National Institutes of Health (Library of Congress Control Number 2010940400, revised 2011).

\section{Whole cortex RNA extraction and RNA sequencing}

For whole cortex analysis of gene expression, TgCRND8 mice were terminally sacrificed using $\mathrm{CO}_{2}$ at $1.5(n=4), 3$ $(n=6), 4.5(n=3) 6(n=5)$, or 10 months $(n=7)$ of age. Corresponding WT littermates were also sacrificed at 1.5 $(n=6), 3(n=6), 4.5(n=4) 6(n=5)$, or 10 months $(n=6)$ of age. Tg2576 mice and corresponding WT controls were sacrificed similarly at $3,6,9,12$, and 15 months of age $(n=$ 4 per group/age). After decapitation, the cortex was removed and rapidly frozen in liquid nitrogen and stored at - 80 until processing. RNA was extracted from TgCRND8 and corresponding control animals using Qiagen RNeasy Mini kit (Qiagen). The Magmax 96 for Microarray RNA extraction kit (Life Technologies) was used to for Tg2576 and corresponding control animals. RNA quality in all samples was assessed with the Agilent2100 Bioanalyzer. Libraries for RNA sequencing were prepared by Beijing Genomics Institute (BGI, Philadelphia, PA) using $100 \mathrm{ng}$ of purified RNA and the Truseq stranded total RNA RiboZero library preparation kit (Illumina) strictly following the Illumina guide (15031048 E?). Briefly, rRNA was depleted with rRNA Removal Mix (RRM) and then fragmented into $160 \mathrm{bp}$ fragments. rRNA-depleted RNA fragments served as templates for first-strand cDNA synthesis using random hexamer-primers, followed by second stand synthesis with the addition of buffer, dNTPs, RNase $\mathrm{H}$, and DNA polymerase I. Double-stranded cDNA was purified using the QiaQuick PCR extraction kit (Qiagen) followed by end repair, base A addition, and ligation of sequencing adapters. Ligated fragments were purified by magnetic beads and amplified via PCR. The resulting library products quantified with the Agilent2100 bioanalyzer and were sequenced using an Illumina HiSeqTM 4000 for a total of 3GB of $50 \mathrm{bp}$ paired-end read data per sample.

\section{Thio-S stain and plaque laser capture}

Fresh frozen TgCRND8 brains ( $n=12 ; 6$ months old) were sectioned axially $(12 \mu \mathrm{m})$ and freeze mounted onto PEN-membrane slides (Applied Biosystems). Slides were kept on dry ice until fixation in $75 \%$ ethanol for $30 \mathrm{~min}$ at room temperature followed by $5 \mathrm{~min}$ incubation in a $1 \%$ Thioflavin-S solution (Sigma). Sections were then dehydrated in $70 \%$ ethanol for $5 \mathrm{~min}, 90 \%$ ethanol for $2 \mathrm{~min}$, and 100\% ethanol for 2 min. Slides were air-dried and stored at room temperature (RT) in a desiccator until LCM. Sections were cut on the laser capture system within $24 \mathrm{~h}$ of staining. An Arcturus laser capture system was used to gather plaque areas (defined as tissue area extending $\sim 100 \mu \mathrm{m}$ beyond the perimeter of the Thio-S positive plaque) and non-plaque areas from TgCRND8 tissue sections. For each TgCRND8 sample, $127 \pm 50$ plaque areas were isolated, lifted onto Arcturus caps, and processed according to manufacturer's directions using a PicoPure Arcturus RNA extraction kit (Applied Biosystems). Non-plaque areas were gathered from the remaining Thio-S negative tissue parenchyma.

\section{In situ hybridization/immunohistochemistry co-labeling} TgCRND8 ( $n=4,35$ weeks old) brains were drop fixed in $10 \%$ buffered formalin for $24 \mathrm{~h}$ at RT prior to processing and embedding in paraffin. Coronal sections ( $5 \mu \mathrm{m}$ thick) were collected on a rotary microtome and mounted onto superfrost $^{\mathrm{TM}}$ plus slides. Trem 2 and CD33 messenger ribonucleic acid (mRNA) were labeled in the formalin-fixed paraffin-embedded TgCRND8 sections using RNAscope $\mathrm{VS}^{\circledast}$ (Advanced Cell Diagnostics, Inc., Hayward, CA) technology. Sections were loaded onto a Discovery Ultra (Ventana Medical Systems, Tucson, AZ) immunostainer, where slides were deparaffinized and treated with protease according to manufacturer's recommendations. Trem2- or CD33-specific mRNA target probes were provided by the manufacturer (Trem2: gene ID 83433 targeted bps 2 through 1081 of the complementary deoxyribonucleic acid (cDNA) sequence, CD33: gene ID 12489 targeted 448 through 1408 of the cDNA sequence). Following signal amplification, target mRNA was visualized with 3,3'-diaminobenzidine (DAB) substrate. A hydrogen peroxide quenching step was performed to prepare the samples for $\beta$-amyloid co-labeling. An anti- $\beta$-amyloid antibody (6E10, Biolegend) was diluted in $10 \%$ goat serum to a working concentration of $0.1 \mu \mathrm{g} / \mathrm{ml}$ to label amyloid plaques. Following incubation of the primary antibody, Discovery Omnimap-Mouse HRP ${ }^{\oplus}$ secondary antibody was applied to the sections. Ventana's Discovery Purple Kit ${ }^{\circledR}$ was used 
to contrast the brown ISH signal. After staining, sections were counterstained with hematoxylin. Sections were scanned with an Aperio XT slide scanner (Leica Biosystems) at $\times 40$ magnification.

\section{NanoString RNA counting methods}

NanoString nCounter technology (http://www.nanostring.com/) allows expression analysis of multiple genes from a single sample. We performed nCounter multiplexed target profiling of 400 custom transcripts. The nCounter codesets were selected prior to the RNAseq data; however, significant representation of differentially expressed genes in the whole cortex and LCM datasets were captured by the custom chips. Fifty nanograms of total RNA per sample was used in all described nCounter analyses according to the manufacturer's suggested protocol. Raw counts for key targets are illustrated in Additional file 1: Figure S1 confirming the RNAseq data via an alternative gene expression profiling platform. Additionally, in Additional file 2: Figure S2, the fold-change data for each transcript on the custom NanoString panel is plotted versus the RNAseq data, for both the whole cortex samples and LCM samples.

\section{RNA sequencing of $L C M$ samples}

Purified RNA (1 ng/sample) from 24 LCM samples (12 transgenic plaque region and 12 transgenic distal non-plaque region) were used for cDNA production using a SMARTer Ultra Low RNA Kit (Clontech, Mountain View, CA) following the manufacturer's instructions with minor modifications. After cDNA amplification and purification, half of the resulting cDNA was used as input for the Nextera XT DNA Sample Preparation Kit (Illumina, San Diego, CA) using a Nextera XT Index Kit (Illumina). Nextera XT sample preparation uses an engineered transposome to fragment and attach sequencing adapters to DNA samples. Illumina Nextera XT protocols were followed for library preparation with 1.0 volumes of purification Agentcourt Ampure XP beads (Beckman Coulter, Beverly MA). No normalization was performed on individual libraries. The library quality was determined using a NanoDrop 1000 (Thermo Scientific, West Palm Beach, FL) to measure library concentration and Bioanalyzer 2100 (Agilent Technologies, Santa Clara, CA) with a DNA 1000 chip to assess library size and quality. Libraries were pooled, and sequencing was performed using an Illumina HiSeq 2500 (Illumina) sequencer with Rapid paired-end sequencing of $76 \times$ 76 bases. Fastq files were generated and demultiplexed using the bcl2fastq version 1.8.4 program.

\section{Data processing}

Alignment and differential gene expression analysis was performed in Omicsoft Array Studio version 7.2.2.29. Briefly, cleaned reads were aligned to the human B37.3 genome reference using the Omicsoft Aligner with a maximum of four allowed mismatches. Gene level counts were determined by the RSEM algorithm as implemented in Omicsoft Array Studio and using RefGene transcript annotation prepared on March 21, 2014. Resultant FPKM data was subsequently ratioed to the average of the WT plaque samples for each gene prior to analysis. Only genes with $\geq 10$ counts in $50 \%$ of the samples in at least one treatment group where considered detected and included in analysis.

\section{Statistical analysis}

Differentially expressed genes were identified by Pearson correlation or $t$ test using MatLab R2010b (Mathworks). A $p$ value cutoff of $<0.001$ was used to identify differentially expressed genes. The FDR corresponding to this $p$ value is given in each of the comparisons to convey relative signature confidence. Set annotation analysis was performed by comparing input sets to GeneGo (www.genego.com), Ingenuity (www.ingenuity.com), and KEGG (www.genome.jp/kegg/) pathway sets. Bonferroni-corrected hypergeometric $p$ values (expectation (e) values) of less than 0.1 were considered significant overlap between sets. Heatmaps were generated in MatLab R2010b using agglomerative clustering.

\section{Results}

\section{Age- and strain-related transcriptomic changes in AD} models

First, we carried out RNAseq on cortexes isolated from Tg2576, TgCRND8, and WT controls. Data revealed a genotype-dependent increase in gene expression differences with respect to age primarily in TgCRND8 animals (Additional file 3: Table S1). In WT animals, relatively few genes showed age-dependent changes. Only 28 genes had Pearson correlation coefficient $(|\rho|)>0.75$ with respect to age in 3-15 month Tg2576 littermate controls, while just 112 genes correlated similarly with age in 1.5-10 month TgCRND8 littermate controls. Similarly, 42 genes had $|\rho|>0.75$ with age in 3-15 month Tg2576 animals. In contrast, 1994 genes had correlation $|\rho|>0.75$ with age in 1.510 month TgCRND8 animals. In order to focus on genotype-related differences without confounding normal aging signatures, we compared the expression differences in Tg2576 and TgCRND8 animals to their respective age-matched WT controls. A small number of genes were differentially expressed between $\mathrm{Tg} 2576$ and WT at all ages examined (Fig. 1a, b). Specifically, $43,42,86,11$, and 58 out of 15,857 detected genes were significantly different $(p<0.001)$ between WT and $\operatorname{Tg} 2576$ animals at 3, 6, 9, 12, and 15 months, respectively (FDR $<37,38,18,100$, and $27 \%$, respectively). Combined, 235 genes displayed a significant 

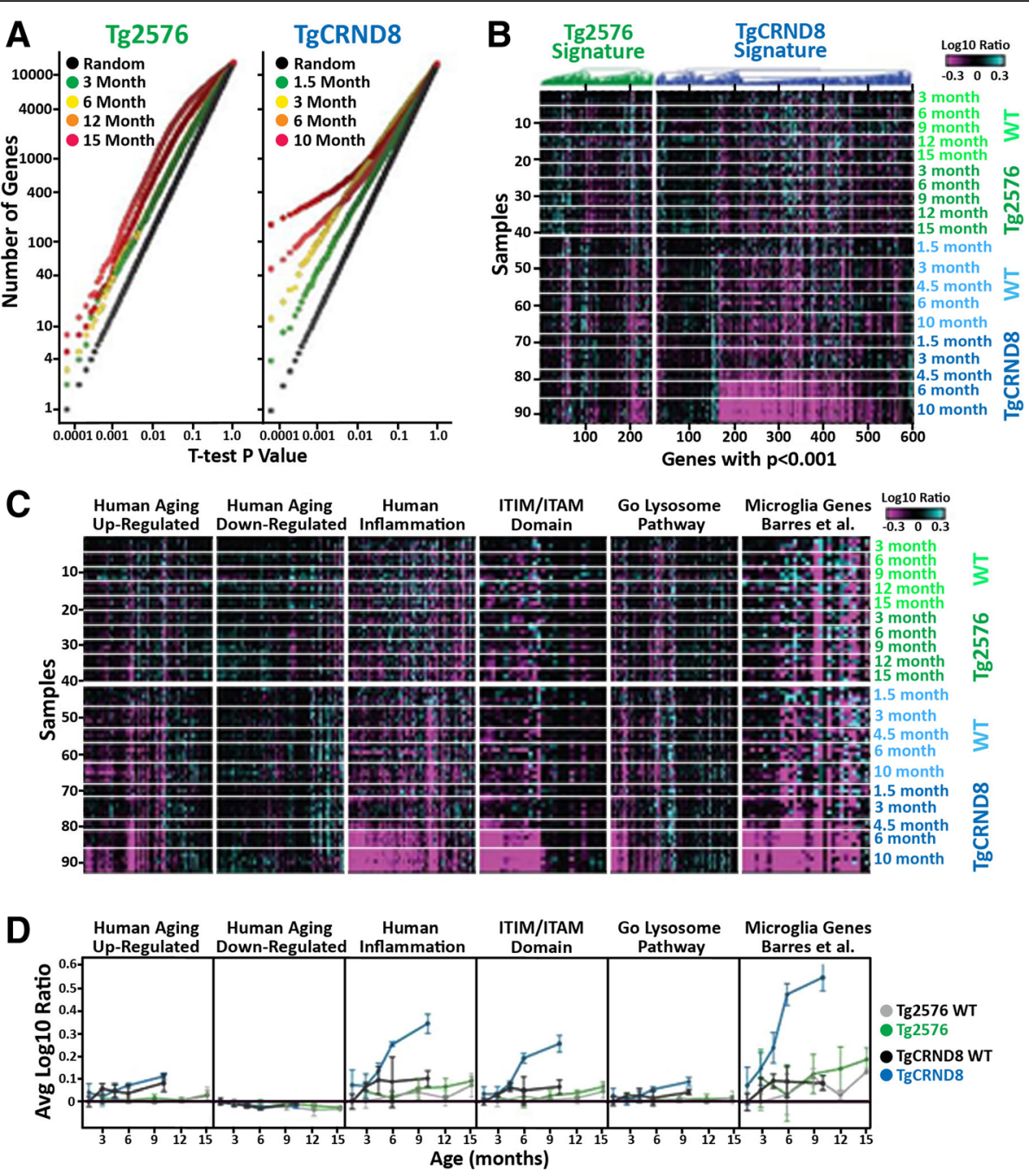

Fig. 1 Transcriptional changes in aging Tg2576 and TgCRND8 brain. a Student's $t$ test $p$ value distributions for gene expression differences between WT and Tg2576 (left plot) or TgCRND8 (right plot) at different ages. Gray line indicates expected false discovery rate (FDR) given multiple test comparisons. $\mathbf{b}$ Heatmap showing $\log _{10}$ ratio values from each sample ( $y$-axis) for each gene ( $x$-axis) with $t$ test $p<0.001$ between Tg2576 (green) and TgCRND8 (blue) versus WT littermate controls at one or more ages. Samples are ordered manually by genotype as indicated. Genes are ordered by test and agglomerative clustering. $\mathbf{c}$ Heatmaps showing $\log _{10}$ ratio values from each sample ( $y$-axis) for each gene ( $x$-axis) within the indicated gene sets. Samples are ordered manually by genotype as indicated. Genes are ordered by agglomerative clustering within each set. $\mathbf{d}$ Signature scores (average of gene values in $\mathbf{C} \pm$ standard deviation) for the indicated gene sets over age in Tg2576 (green) and WT littermate control (gray) as well as TgCRND8 (blue) and WT littermate controls (black)

difference $(p<0.001)$ between $\operatorname{Tg} 2576$ and WT at one or more ages tested, 125 genes (53\%) of which were upregulated. However, a strong progression of genotype-related expression changes was observed in TgCRND8 animals (Fig. 1a, b). At 1.5 months of age, 52 genes had significantly different expression in the TgCRND8 cortex compared to WT controls $(p<0.001$, FDR $<29 \%$ ); by 3 months of age, 108 genes (FDR < $14 \%)$ were similarly regulated and this number increased to $170(\mathrm{FDR}<9 \%)$ and $351(\mathrm{FDR}<4 \%)$ at 6 and 10 months, respectively. Combined, 582 genes displayed a significant difference $(p<0.001)$ between
TgCRND8 and WT at one or more ages tested, 464 $(80 \%)$ of which were upregulated. Numerous gene pathways were significantly (Bonferroni-corrected $p$ value $(e)<0.1)$ enriched among the genes upregulated in TgCRND8 cortex, predominantly those associated with immunity inflammation lipid metabolism and protein salvage (Additional file 4: Table S2). For example, numerous lysosomal and ITIM (immunoreceptor tyrosine-based inhibition motif; S/I/V/LxYxxI/V/L) and ITAMs (immunoreceptor tyrosine-based activation motif; consensus sequence YxxI/Lx6-12YxxI/L) signaling genes exhibited age-dependent increases in 
expression in TgCRND8 mice (Fig. 1c, d). However, no biological pathways were identified as significantly enriched among the smaller number of genes downregulated in $\mathrm{Tg} 2576$ or in either of the upregulated or downregulated gene sets in Tg2576 despite Tg2576 mice presenting with plaque pathology (Additional file 5: Figure S3).

\section{Translational transcriptomic profiling}

Next, we assessed whether orthologs of the previously identified human cortical gene networks impacted with aging and $\mathrm{AD}$ were modulated with age in the mouse models examined (Additional file 6: Table S3) [34]. As expected, little progression of the human aging signatures was observed in mouse cortex across the ages examined (Fig. 1c, d). Although some of the aging signature genes changed significantly, the trends across all genes with age and the differences between $\mathrm{Tg} 2576 / \mathrm{TgCRND} 8$ and WT controls were not significant (Fig. 1c, d). The human inflammatory signature was upregulated in aged specimens. This signature was robustly potentiated in AD subjects regardless of age and also exhibited strong progression with TgCRND8 aged animals. Indeed, this signature was highly enriched $(p<3 e-53)$ among the 464 genes differentially upregulated $(p<0.001)$ between TgCRND8 and WT controls (Fig. 1). Similarly, we examined whether the brain cell-type specific gene sets identified by Zhang et al., 2013 were enriched among the up or down arms of the Tg2576 or TgCRND8 signatures. Significant overlap was only observed with the microglial gene set, which was highly enriched $(p<6 e-24)$ among the genes upregulated in TgCRND8 (Fig. 1c, d) [23].

\section{Plaque transcriptomic signature}

In order to establish that the identified gene networks, particularly the human-related disease signatures, were not only temporally but also regionally associated with $\mathrm{A} \beta$ pathology LCM was performed around Thio-S-labeled sites. We used the in situ hybridization pattern of TREM2 and CD33 around A $\beta$ plaques in the aged TgCRND8 mouse (Fig. 2) to qualitatively define the radius of the laser capture parameters. Representative images were stained with Iba-1 and 6E10 (Fig. 2c, d) to illustrate plaque and non-plaque areas processed for RNA seq. Brain slices prepared from aged TgCRND8 mice and processed for $A \beta$ immunohistochemistry (6E10 labeling, magenta) combined with TREM2 (panel A) or CD33 (panel B) in situ hybridization via RNAScope (brown) confirmed their associated expression pattern with $\mathrm{A} \beta$ pathology in the TgCRND8 model and supported our rationale for laser capture microdissection not just of Thio-S-labeled plaques (as performed by [35]) but also the surrounding penumbra (Fig. 2). RNAseq of laser capture samples revealed transcriptomic differences between plaque niche and non-plaque TgCRND8 tissue. In TgCRND8 mice, 82 out of 12,461 detected genes were significantly $(p<0.001$; FDR $<15 \%)$ regulated compared to non-plaque TgCRND8 tissue; 67 of these genes were upregulated and 15 were downregulated (Fig. 3a, b; Additional file 7: Table S4). Numerous gene pathways were significantly $(e<0.1)$ enriched among the genes upregulated in the plaque niche, predominantly those associated with vesicles, immunity, inflammation, and protein salvage, for example lysosomal or immunoreceptor tyrosine-based activation/inhibition motif (ITIM/ITAM) signaling genes (Fig. 3c, Additional file 4: Table S2). As in the TgCRND8 versus WT comparison, the human inflammation signature $(p<3 e-34)$ as well as the microglial-specific signature $(p<$ $7 e-7)$ were significantly enriched among the genes upregulated within the plaque niche signature, while the other human and cell-type gene sets were not (Fig. 3c). The expression differences in TgCRND8 mice and those enriched in the plaque niche were highly overlapping. The majority of the genes upregulated in TgCRND8 cortex compared to WT animals were also expressed higher in CRND8 plaque versus non-plaque (Fig. 3a). Conversely, the majority of the genes upregulated in the plaque versus non-plaque comparison were also upregulated in 610-month-old TgCRND8 animals compare to WT (Fig. 4b). Several genes $(12,130)$ were detected in both experiments, including 342 genes that were upregulated in TgCRND8 vs WT cortex $(p<0.001$ at any age) and 67 genes upregulated in plaque vs non-plaque $(p<0.001)$, of which 46 were overlapping between both signature sets $(p<2 e-56)$ (Fig. 4c). We confirmed the findings from our RNAseq data using a customized NanoString nChip showing that key plaque-associated genes were upregulated (Trem2, Tyrobp, Cd68, Clec7a, Tspo, Itgfax) (Additional file 1: Figure S1). Data were analyzed for expression of a set of markers associated with resident microglia or peripheral macrophages as outlined by Hickman et al. to characterize the phenotype of cells surrounding the plaque [36]. Results show that 7 out of the top 25 most abundant genes in resident microglia were significantly upregulated in the plaque niche compared to non-plaque samples (Additional file 8: Figure S4). Conversely, only 1 gene associated with peripheral macrophage expression was significantly upregulated in the plaque niche, Complement C4-b (C4b) (Additional file 8: Figure S4). This phenotypic analysis was more pronounced in whole cortex samples resulting in 13 out of the top 25 microglial-specific genes were significantly upregulated in TgCRND8 mice compared to WT compared to only 1 gene in the peripheral macrophage signature (Additional file 8: Figure S4).

To further characterize the microglia phenotype observed in the TgCRND8 plaque niche, we compared the gene signatures identified here to those of the microglial subtype, disease-associated microglia (DAM), recently shown to surround plaques in another mouse $\mathrm{AD}$ model expressing five familiar AD mutations (5XFAD) [37]. 

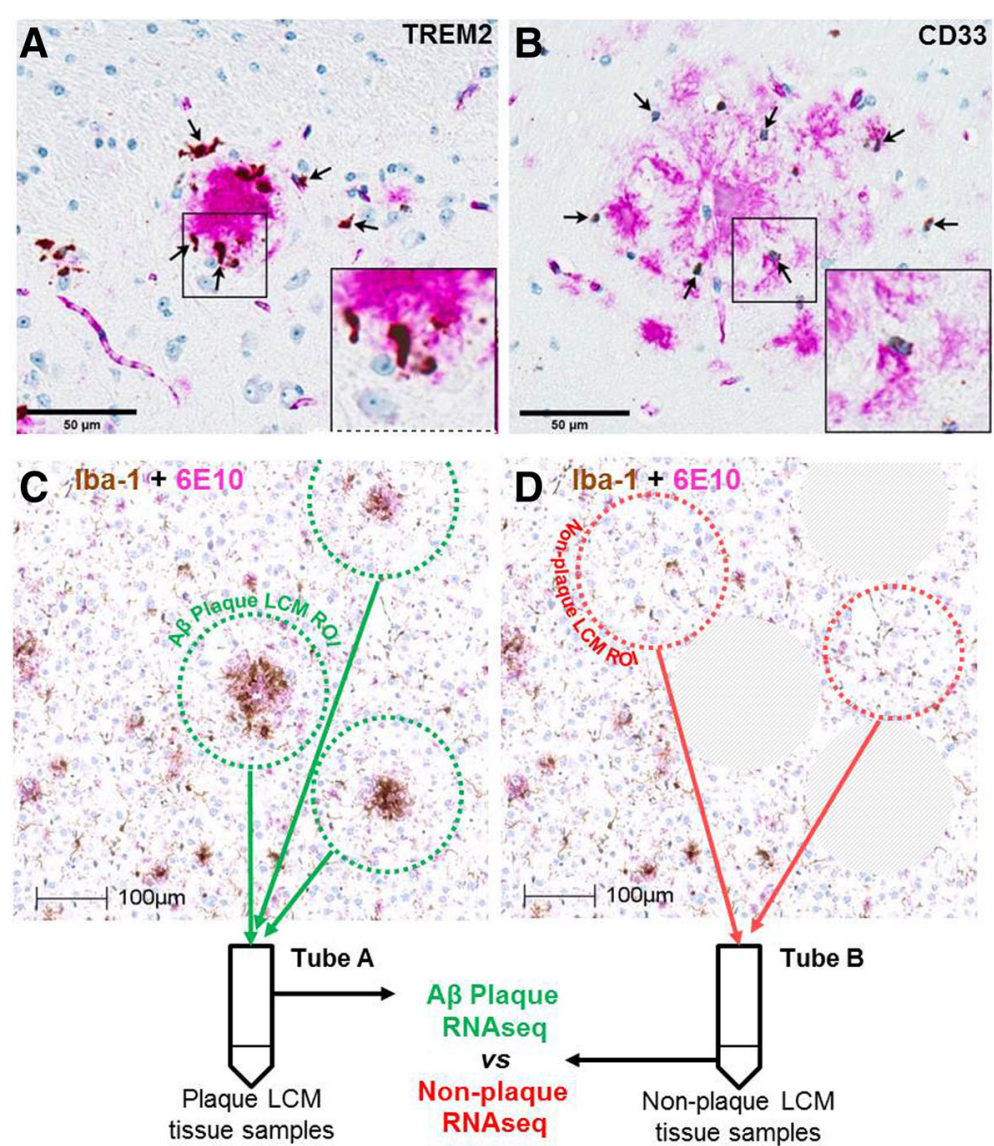

Fig. 2 Localization of TREM2 and Cd33 around amyloid-beta (Aß) plaques in the aged TgCRND8 mouse. Brain slices were prepared from 35week-old wild-type and TgCRND8 mice and processed for amyloid-beta immunohistochemistry (6E10 labeling, magenta) combined with TREM2 (panel a) or CD33 (panel b) in situ hybridization via RNAScope (brown). Visualization of TREM2 and Cd33 confirms their associated expression pattern with $A B$ pathology, supporting the rationale for laser capture microdissection of Thio-S-labeled plaques for transcriptome-wide RNA sequencing. Representative images show section sampling for LCM and RNA seq (c, d). Images show Iba1 (brown) and 6E10 (magenta) immunostainings. Scale bars, 50 and $100 \mu \mathrm{m}$

Indeed, genes reported to be expressed higher in the DAM population ( $>1.5$ fold, $p<1 e-5$ ) were strongly enriched among the upregulated gene signatures in both the TgCRND8 whole cortex and the LCM-dissected TgCRND8 plaque tissue signatures $(p=4 e-25$ and $p=8 e-13$, respectively) (Fig. 5a-d; Additional file 4: Table S2, Additional file 6: Table S3, and Additional file 7: Table S4). Across all three gene sets, eight genes were commonly expressed: $\mathrm{Ccl} 6$, Cd9, Ctsz, Gusb, Lyr2, Npc2, Trem2, and Tyrobp. In contrast, the DAM marker genes were not significantly represented among the Tg2576-related signatures $(p=0.3)$ (Fig. 5a, c). In additional analyses, expression of markers of M1 and M2 microglia as outlined in [38,39] were also analyzed for differential expression in plaque vs. non-plaque samples as well as in whole cortex samples from TgCRND8 compared to WT controls. Results show a significant increase in expression of only one marker of M1 activation, $C D 16 a$, in the plaque niche compared to non-plaque controls $(3.06-$ fold; $p<0.0001)$. No markers of
M2 activation were significantly upregulated in plaque samples compared to non-plaque. Whole cortex TgCRND8 displayed significant $(p<0.001)$ expression of four markers of M1 activation, CXCL10 (34.52-fold; $p<0.0001$ ), CCR5 (1.51-fold; $p<0.0001), C D 86$ (2.25-fold; $p<0.0001)$, and CD16a (2.62-fold; $p<0.0001)$ in TgCNRD8 compared to WT, and only two markers of M2 activation/repair, Clec7a (72.33-fold; $p<0.0001)$, and TGFB (2.23-fold; $p<0.0001)$.

\section{Discussion}

Many studies have demonstrated a strong association between inflammation, innate immunity, and amyloid plaques in both human AD and preclinical models [23, 40]. Data described here extend and deepen prior characterizations of two different APP transgenic mouse models of AD, the $\operatorname{Tg} 2576$ and TgCRND8 lines, by leveraging next-generation RNA sequencing to enable comprehensive and progressive transcriptional profiling of cortical $A \beta$ plaque pathology. Data also provide context for microglial reactivity in the 


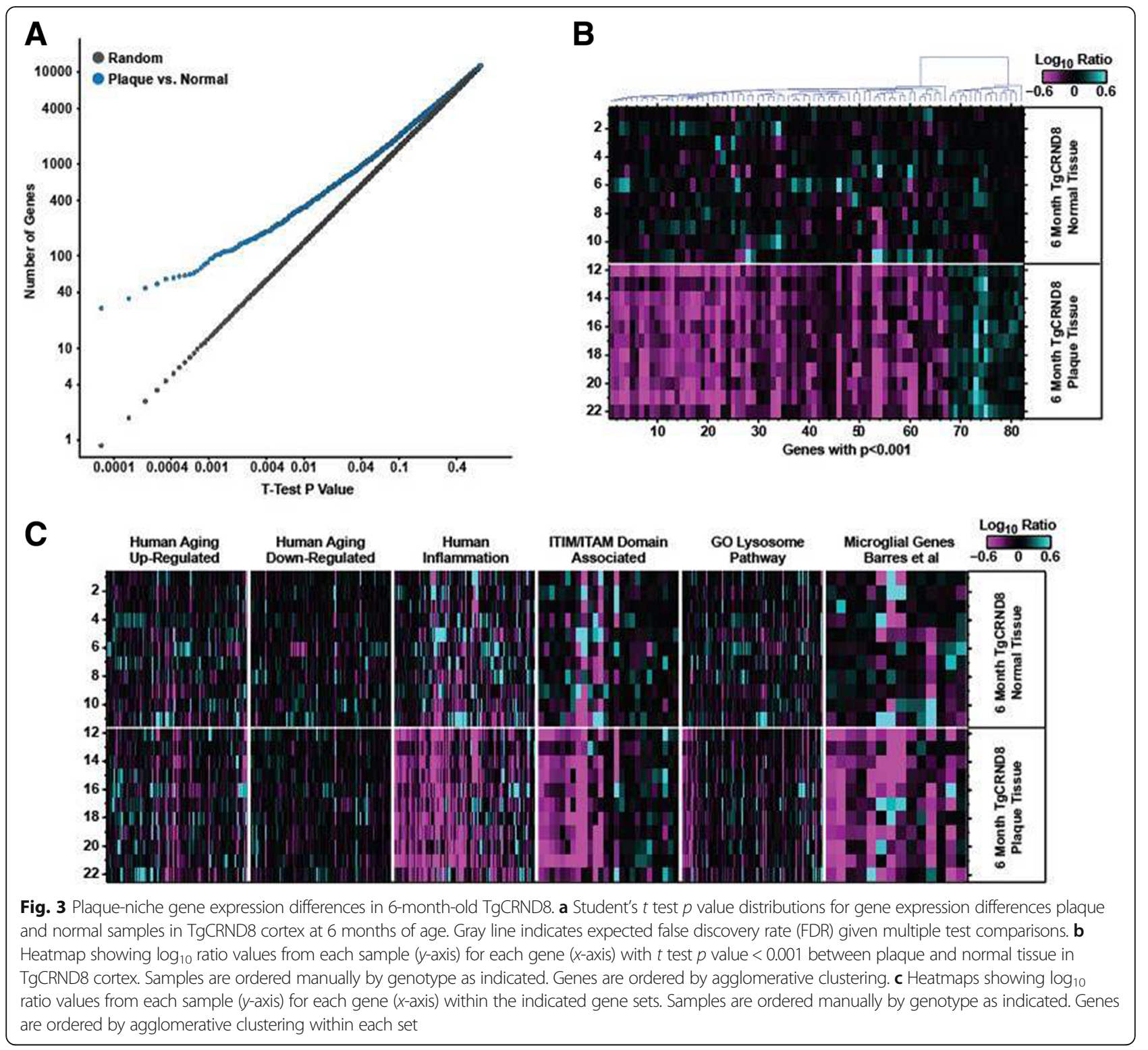

plaque niche; the majority of transcriptional changes observed in aging TgCRND8 cortical brain homogenates were specifically enriched within the plaque niche compared to samples from non-plaque regions of the cortex from TgCRND8 mice. These data provide crucial context for translational relevance of two mouse models of $\mathrm{AD}$ and insights into transcriptional regulation in the plaque niche.

The Tg2576 mouse overexpresses a variant form of APP harboring dual Swedish mutations (KM670/671NL) under the control of the hamster PrP promoter, resulting in substitutions of two amino acids, lysine $(\mathrm{K})$ and methionine $(\mathrm{M})$ to asparagine $(\mathrm{N})$ and leucine $(\mathrm{L})$ [41-43]. In vitro, this mutation results in elevated production and secretion of both $\mathrm{A} \beta 40$ and $\mathrm{A} \beta 42$, as this mutation lies immediately adjacent to the $\beta$-secretase site on APP resulting in higher affinity to BACE1 compared to wild-type APP [43]. The
Swedish mutation does not generally lead to amyloidosis by shifting the ratio of $A \beta 42 / A \beta 40$. The Swedish mutation predominantly leads to the secretion of $A \beta 40$, a less amyloidogenic peptide. Similarly, the TgCRND8 mouse also overexpresses the Swedish APP mutation under control of the hamster PrP promoter but additionally contains the Indiana (V717F) mutation in the human APP transgene. In contrast to $\mathrm{AD}$-associated residues modulating $\beta$-secretasemediated cleavage of APP, pathogenic mutations such as V717F occurring at the $\Upsilon$-secretase processing site of APP increase the ratio of $A \beta 42 / A \beta 40$, lead to elevated secretion of the intrinsically aggregation prone $A \beta 42$, and increase the ratio of $A \beta 42 / A \beta 40$. It is widely accepted that $A \beta 42$ is the major component of amyloid plaques in $\mathrm{AD}$ brain, despite observations that the concentration of $A \beta 40$ is significantly higher than $A \beta 42$. In vitro, $A \beta 40$ and $A \beta 42$ are 


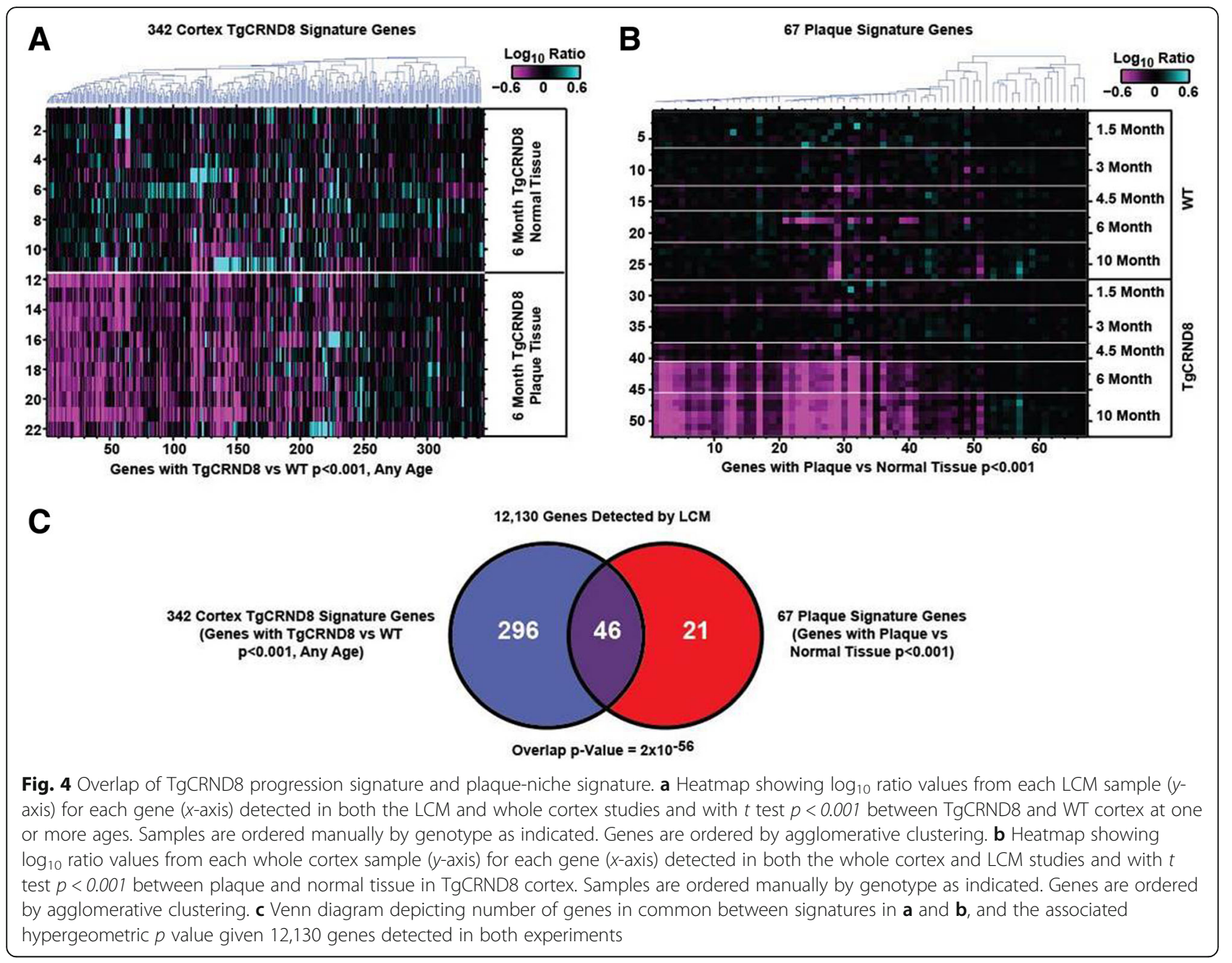

capable of forming interlaced fibrils [44]; however, the rate of aggregation is controlled in part by the ratio of $A \beta 40$ with $A \beta 42$, as peptide mixtures are less amyloidogenic than A $\beta 42$ alone [45-49]. In line with this view, transgenic mice expressing solely $\mathrm{A} \beta 40$ do not develop amyloid pathology or form insoluble aggregates [50]. Similarly aligned, the Tg2576 model exhibits slower $A \beta$ pathology compared to TgCRND8 mice, with the extent of insoluble $A \beta$ levels being more severe in TgCRND8. Here, we exploit these fundamental differences between two APP models to broadly evaluate how that impacts secondary responses to $A \beta$ plaque burden by whole transcriptome sequencing.

Our findings show that the human AD neuroimmune signature develops progressively in the TgCRND8 mouse cortex, coincident with known trajectories of $A \beta$ deposition, but not in the $\mathrm{Tg} 2576$ mouse, raising intriguing questions about how the brain responds to different composition states of $A \beta$. The signature changes observed in brain homogenates highly overlap with the local expression profile of the plaque niche. One possibility is the degree of insoluble $A \beta$ aggregate burden directly triggers the transcriptional changes observed in the TgCRND8. However, it is more likely that a constellation of contributing neurodegenerative triggers occur within the plaque-laden niche. For example, alterations in synaptic density, the extracellular matrix proteome (i.e., matrisome), the local secretome, and possibly changes in lipid composition may vary with specific $A \beta$ plaque pathology [48]. Future in vitro studies exploring whether exposure to monomeric, oligomeric, and fibrillar $A \beta$ species which are sufficient to induce the observed transcriptional responses would help to address the specificity and complexity of the sequencing changes observed here via laser capture of $A \beta$ plaques.

A similar study utilizing LCM to isolate protein-rich amyloid plaques in the absence of surrounding cellular penumbra from two clinical AD cases previously reported an enrichment of glial fibrillary acidic protein (GFAP), a marker for reactive astrocytes, and vimentin, an intermediate filament, in Thio-S positive plaques in AD patients [35]. In this proteomic study, several subunits of lysosomal ATPase were concentrated in the plaque, along with other 


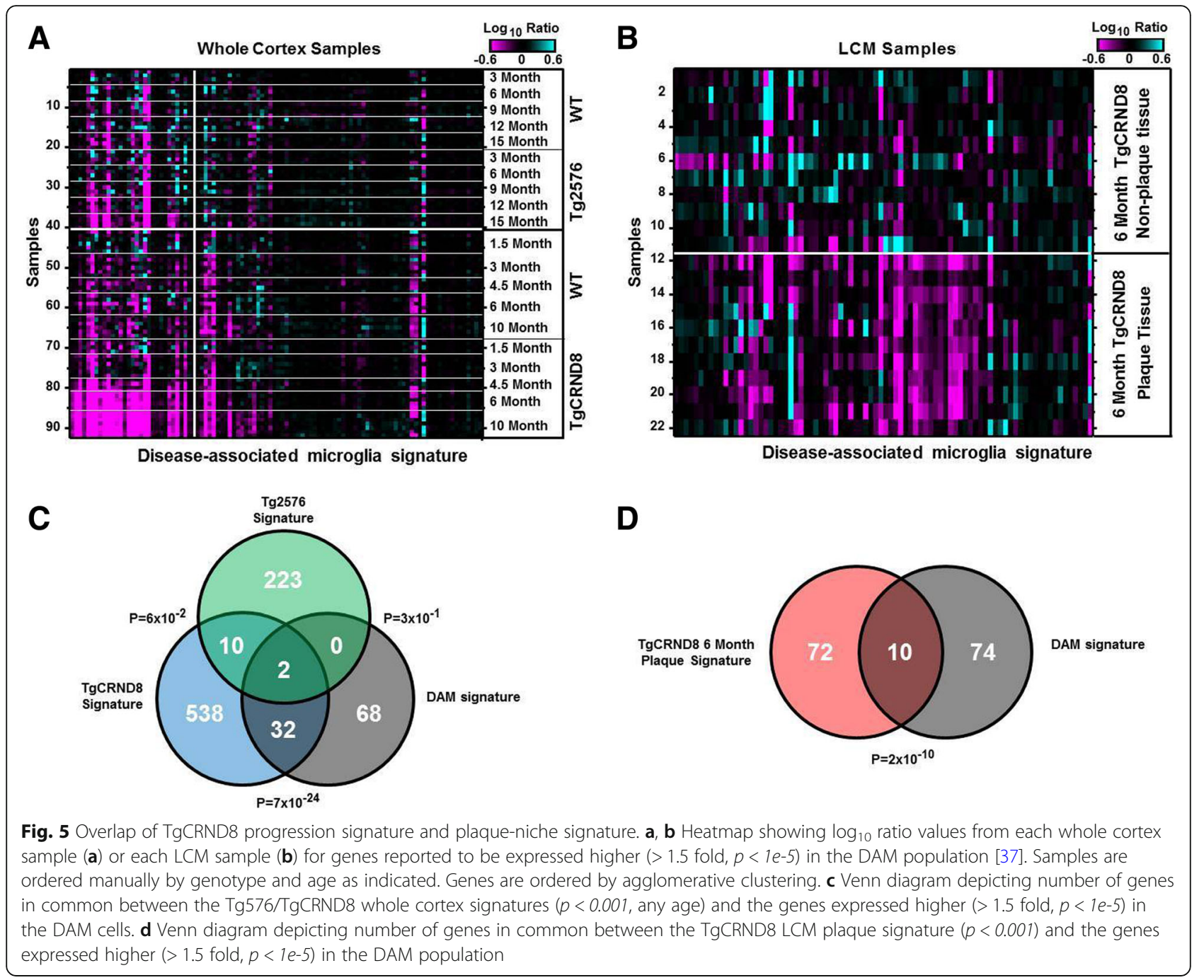

markers of the ubiquitin-proteasome system, collectively indicating an activation of protein degradation mechanisms. Liao et al. also identified many heat shock proteins, specifically HSP90, enriched in the plaques [35]. Our current RNAseq data also demonstrate a significant $(p<0.001)$ increase of nearly 3 -fold in the expression of GFAP in the plaque niche compared to non-plaque tissue. While reactive astrocytes have been shown to express high levels of heat shock proteins, we did not observe changes in heat shock family transcripts despite evidence of enriching astrocyte-specific markers in the plaque niche of the TgCNRD8 mouse. However, consistent with human proteomic analysis, the current transcriptomic insights presented here (Additional file 4: Table S2) confirm a significant upregulation in the expression of genes associated with lysosomal function in plaque areas compared to non-plaque. The observations of alterations in lysosomal pathways support the notion that the plaque core and its surrounding glia comprise a hot spot for autophagy-lysosomal activity and that pathways related to protein homeostasis and phagocytosis of extracellular amyloid plaques or other neurodegenerative debris are altered in the plaque niche.

Previous histological studies unequivocally validated a discrete regional association between $A \beta$ plaques and microglia in human and mouse models of AD, and current data reveals a complex picture regarding the upregulation of markers of microglia, potentially indicating a phenotypic difference between plaque-associated microglia and non-plaque-associated microglia [51-57]. Not surprisingly, canonical markers of microglia such as fractalkine (cx3cr1), cd68, and itgam (CD11b) were significantly upregulated in the plaque niche compared to non-plaque areas: $2.68,3.0$, and 2.0 -fold, respectively. Significant increases in the expression of $c x 3 c r 1$ and $c d 68$ were also sufficiently robust to detect in whole cortex analysis in 6 -month-old animals $(p<0.001)$; in contrast, CD11b was not significantly upregulated in the whole cortex at this age. Interestingly, the classical marker of activated microglia aif1 (Iba1) demonstrated no significant change between plaque-associated samples and non-plaque tissue. 
However, the longitudinal profiling suggests that AIF1 induction emerges as a later adaptation to amyloid plaques, as it is more highly upregulated in the 10-month-old TgCRND8 cohort (2.1-fold) compared to 6-month TgCRND8 animals (1.5-fold), the age at which the plaque niche was profiled in this laser capture study. These observations serve to highlight that classical markers of microglia phenotypic state carry unique time-courses and regional-specificity which should be accounted for when utilizing a single or limited subset of cell-specific markers to draw inferences on neuroimmune status, responses to treatment interventions, and consequent interpretations on efficacy of interventions.

Another common method to evaluate microglial states has been through more extensive marker classifications adopted from peripheral macrophage profiling in order to draw conclusions regarding the M1 vs M2 polarization status of resident CNS microglia [58]. According to this paradigm, M1 microglia are associated with the production of pro-inflammatory cytokines such as tumor necrosis factor- $\alpha$ (TNF $\alpha)$, interleukin- $1 \beta$ $(I L-1 \beta)$, and interleukin-6 (IL-6), as well as the catalysis of nitric oxide $(\mathrm{NO})$ from arginine and release of various state-specific chemokines. Alternatively, markers of the M2 activation and repair state include YM1 (Chi3l3), non-TLR pattern recognition receptors such as dectin-1 (Clec7a), CD200R, and MRS1, along with Arg1 which favors arginine metabolism, and ALOX12 and 15 involved in arachidonic acid metabolism. In the current study, we did not observe a clear distinction of the M1 versus M2 phenotypes of microglia across broader panels; either in whole tissue comparisons to WT mice or focally around the $A \beta$ plaque niche within the TgCRND8 mouse. Microglial functions are incredibly diverse and comprise a broad spectrum of adaptations from inflammation, neuroprotection, and neuroimmune processes including phagocytosis, clearance of cellular debris, expression of growth factors, production and release of cytokines, and synaptic pruning [59]. Possible explanations for a failure to see clear activation of an M1 or M2 phenotype include extreme heterogeneity of microglial phenotypes, the stage of TgCRND8 plaque progression in the current study, or the relative absence of other cardinal features of $\mathrm{AD}$ such as tau pathology or robust neuronal loss.

In recent years, microglial research has shifted away from the M1/M2 nomenclature, with the aid of tools such as specific microglia antibody-labeling and single-cell transcriptomics and proteomics analysis. Using these tools, research groups have been able to identify the microglial transcriptome in neurodegenerative states that are more diverse than M1/M2 polarization which is more phenotypic relevant. In one such study, using an AD model, disease-associated microglia or "DAM" cells surround the plaques and exhibit distinct genetic profiles that differ from homeostatic/non-disease-associated microglia [37]. We carried out comparative analyses between published DAM signature profiles and our data sets which revealed that DAM-signatures are present in the plaque-associated tissue in the TgCRND8 mice and this also correlates with age. From this gene set, 32 genes overlapped between TgCRND8 plaque niche versus DAM genes, with two of these genes being Tyr$o b p$ and Trem2. There were no genes overlapping in Tg2576 versus the DAM signature (Fig. 5c). Thus, our data suggests that the TgCRND8 model is a more relevant model to understand polarization states and plaque niche.

The TgCRND8 transcriptional profiles align with previous findings of robust transcriptional responses in recently discovered genetic links between microglia, innate immunity, and AD. Numerous GWAS and meta-analyses in various AD cohorts' studies have identified novel microglialassociated receptors TREM2 and CD33 for $\mathrm{AD}[16,18,29]$. This has naturally led to investigations of their roles in $\mathrm{AD}$ in preclinical models. In the current study, regional assessment of the $A \beta$ plaque niche revealed TREM2 and its signaling adaptor TYROBP (which encodes the DAP12 protein) are significantly $(p<0.001)$ upregulated in the $A \beta$ plaque niche compared to non-plaque areas. Both markers show a 3-fold increase in expression near the plaque compared to non-plaque samples. An expanded view beyond these hub genes confirms that several ITIM/ITAM-associated transcripts are also enriched around plaques. In TgCRND8 cortical homogenates, this innate immune panel was shown to be upregulated at 6 and 10 months of age, as well. It is important to highlight, however, that the ITIM/ ITAM-associated module is not invariably associated with A $\beta$ plaque pathology. In the Tg2576 model, we did not observe significant induction of this AD-implicated innate immune signature. Therefore, when juxtaposed with robust progressive signature induction in the TgCNRD8 mice, it is clear that not all mouse APP models are equivalent in this regard.

Exploring more closely specific ITIM/ITAM-associated changes, we identified novel dysregulated pathway nodes as potential modulatory factors, including genes known to be enriched in microglia such as receptor and non-receptor tyrosine kinases, as well as associated phosphatases predicted to inhibit phagocytic signal transduction. For instance, it is widely known that DAP12 signals via SYK induction and its downstream signaling may be negatively regulated by various phosphatases such as PTPN6 and INPP5D. Interestingly, not only do we observe robust SYK upregulation around amyloid plaques ( $>3$-fold) but associated protein tyrosine phosphatases PTPN6 and INPP5D 
are also enriched (upregulated 6.0-fold and 2.7-fold respectively). Both PTPN6 and INPP5D are involved in the signaling via inhibitory receptors found in innate immune cells, such as CD33 for example. Moreover, in a meta-analysis of major AD GWAS, the rs35349669 locus on chromosome 2 encoding INPP5D was recently identified as a novel AD risk-associated locus [60-62]. Within the plaque niche, we also observed that several inhibitory ITIM-containing receptors in addition to CD33 are also enriched, such as Slamf9 (7.8-fold, $p=0.011$ ), FCGR1 (2.2-fold, $p=0.002$ ), and LAIR1 (2.0-fold, $p=0.001$ ). Likewise, we identified several additional ITAM-associated receptors such as FCGR1 (2.2-fold, $p=0.002$ ), FCGR3 (3.1-fold, $p=1.4 e-06$ ), and CSF1R (1.5-fold, $p=0.0013$ ) are significantly increased in the plaque niche compared to non-plaque areas.

Functional studies have begun to identify a role for the TREM2/DAP12 pathway in promoting cell survival and phagocytic capacity [63]. TREM2 deficiency reportedly impairs the phagocytosis of apoptotic neurons in microglia [64], possibly through apolipoprotein $\mathrm{E}$ and lipid-sensing mechanisms [65-68]. Similarly, in primary microglia isolated from TREM2 knockout mice, impaired phagocytic capacity of microbeads as well as Escherichia coli bacteria imply that TREM2 plays an important regulatory role in innate immune activity and possibly host defense mechanism [69]. Similar functional insights have been reported for CD33. A study by Gricuic et al. observed increased expression of $\mathrm{CD} 33$ positive microglia in $\mathrm{AD}$ brains, a significant positive correlation between $A \beta$ pathology and CD33 expression as well as an increase in microglial uptake of $A \beta$ in vitro after CD33 inactivation [32]. Interestingly, clinical data show an increase in PiB imaging in patients with the risk allele for CD33 [16], further demonstrating a relationship with amyloid pathology and potential to track the progression of disease-related PET-imaging biomarkers. These findings serve as the foundation for the therapeutic hypothesis that blockade of CD33 function could be a compelling alternative to accelerate $\mathrm{A} \beta$ clearance [32]. Thus, when considering collectively the functional roles for TREM2 and CD33 in microglia and AD, it appears that converging and opposing innate immune regulation may exist and dictate risk status for human $\mathrm{AD}$.

\section{Conclusions}

The current study highlights the importance of careful consideration and selection of appropriate APP mouse models specifically for exploring neuroimmune modulation, in particular ITIM/ITAM-associated mechanisms, such as TREM2 and CD33. Combined with the insights from human genetics, these findings provide broad evidence for the presence of a complex relationship between activating and inhibitory mechanisms which collectively may determine innate immune status around the $A \beta$ plaque niche. Future studies utilizing single-cell profiling of microglia in $\mathrm{AD}$ mouse models to further characterize the co-expression of immune activating (e.g., ITAM) and suppressing (e.g., ITIM) mechanisms and will help to define how the underlying regulation of mechanisms controlling this microglial innate immune rheostat are shaped by neuropathology. Such studies will also help clarify whether distinct heterogeneous sub-populations of microglia exist around amyloid plaques, or whether competing inhibitory and activating nodes are commonly present within individual plaque-associated microglia. The observations provide tissue-level insights into plaque-associated transcriptional signatures to encourage more fine characterization of cell-specific pathway changes across such broad panels of markers.

\section{Additional files}

Additional file 1: Figure S1. Nanostring confirmation of plaque associate selected gene in TgCRND8. Selected plaque associated genes that were upregulated in LCM tissue in TgCRND8 mice by RNAseq were confirmed using a Nanostring customized chip ( $\mathrm{n}=4$ per group). Bar graphs show nCounts (mean \pm s.e.m) to highlight changes for a subset of specific transcripts included for direct comparison. ${ }^{*} p<0.05$, ${ }^{* *} p<0.01$, by student t-test, 2-tailed. (JPG $51 \mathrm{~kb}$ )

Additional file 2: Figure S2. Nanostring confirmation of plaque associate selected gene in TgCRND8. Selected plaque associated genes that were upregulated in LCM tissue in TgCRND8 mice by RNA seq confirmed using Nanostring customized chip ( $n=4$ per group). Dot plots show mRNAs transcripts (mean \pm s.e.m). ${ }^{*} p<0.05,{ }^{* *} p<0.01,{ }^{* * *} p<0.001$, by student t-test, 2 -tailed. (JPG $175 \mathrm{~kb}$ )

Additional file 3: Table S1. Age and strain-related transcriptomic changes in AD models. RNAseq was carried out on cortexes isolated from Tg2576 (ages: $3 \mathrm{mo}, 6 \mathrm{mo}, 9 \mathrm{mo}, 12 \mathrm{mo}$ and $15 \mathrm{mo}$, respectively), TgCRND8 (1.5mo, 3mo, $4.5 \mathrm{mo}, 6 \mathrm{mo}$ and $10 \mathrm{mo}$, respectively) and WT age-matched controls mice. Data shows statistical analysis of age correlation between each strain, $p$-value and $\log 10$ analysis. Differentially expressed genes were identified by Pearson correlation or T-test using Matlab R2010b (Mathworks). A p-value cutoff of $<0.001$ was used to identify differentially expressed genes. The FDR corresponding to this $p$-value is given in each of the comparisons to convey relative signature confidence. Set annotation analysis was performed by comparing input sets to GeneGo (www.genego.com), Ingenuity (www.ingenuity.com) and KEGG (www.genome.jp/kegg/) pathway sets. Bonferroni corrected hypergeometric $p$-values expectation (e)-values) of less than 0.1 were considered significant overlap between sets. (XLS 7280 kb)

Additional file 4: Table S2. Age and strain-related pathway analysis in TgCRND8 cortex. Pathways analysis showing data from upregulated network processes in TgCRND8 cortex and plaque-associated tissue. Differentially expressed genes were identified by Pearson correlation or T-test using Matlab R2010b (Mathworks). A p-value cutoff of $<0.001$ was used to identify differentially expressed genes. The FDR corresponding to this $p$-value is given in each of the comparisons to convey relative signature confidence. Set annotation analysis was performed by comparing input sets to GeneGo (www.genego.com), Ingenuity (www.ingenuity.com) and KEGG (www.genome.jp/kegg/) pathway sets. Bonferroni corrected hypergeometric p-values (expectation (e)-values) of less than 0.1 were considered significant overlap between sets. (XLSX $81 \mathrm{~kb})$

Additional file 5: Figure S3. Tg2576 brain sections illustrating amyloidbeta pathology. Representative images of plaque pathology in 15-month old transgenic mice via anti-amyloid- $\beta, 1-12$ (26D6). (JPG 183 kb)

Additional file 6: Table S3. Human AD translational transcriptomic profiling Comparative gene network analysis was carried out from the mouse models used (tgCRND8 and Tg2576 models) in our study with that of: 1) Human aging (up-regulated) 2) Human aging (down-regulated) 3) Human inflammation 
signature 3) ITIM/ITAM-domain associated network signature 3) Mouse microglial gene signature (Barres et al., 2013) and 4) Mouse disease-associated microglia or DAM gene signature (Keren-Sheul et al., 2017). Differentially expressed genes were identified by Pearson correlation or T-test using Matlab R2010b (Mathworks). A. $p$-value cutoff of $<0.001$ was used to identify differentially expressed genes. The FDR corresponding to this $p$-value is given in each of the comparisons to convey relative signature confidence. Set annotation analysis was performed by comparing input sets to GeneGo (www.genego.com), Ingenuity (www.ingenuity.com) and KEGG (www.genome.jp/kegg/) pathway sets. Bonferroni corrected hypergeometric $p$-values (expectation (e)-values) of less than 0.1 were considered significant overlap between sets. (XLSX $26 \mathrm{~kb}$ )

Additional file 7: Table S4. Laser captured plaque transcriptomic signature. RNAseq data of laser-capture microscopy of TgCRND8 plaques versus non-plaque tissue as outlined in schematic in Fig. 2. Data shows statistical analysis of TgCRND8 plaques versus non-plaque tissue at 6 months of age ( $p$-value) and log 10 analysis of the ratio of TgCRND8 plaques versus non-plaque tissue. Differentially expressed genes were identified by Pearson correlation or T-test using Matlab R2010b (Mathworks). A p-value cutoff of $<0.001$ was used to identify differentially expressed genes. The FDR corresponding to this $p$-value is given in each of the comparisons to convey relative signature confidence. Set annotation analysis was performed by comparing input sets to GeneGo (www.genego.com), Ingenuity (www.ingenuity.com) and KEGG (www.genome.jp/kegg/) pathway sets. Bonferroni corrected hypergeometric $p$-values (expectation (e)-values) of less than 0.1 were considered significant overlap between sets. (XLS $1410 \mathrm{~kb}$ )

Additional file 8: Figure S4. Expression of microglial versus peripheral macrophage markers. Data were analyzed for fold changes in genes associated with either resident microglia (A) or peripheral macrophages (B) as outlined by Hickman et al., 2013. Results demonstrate significant ( $\left.{ }^{*} p<0.001\right)$ expression of 7 out of the top 25 most abundant genes in microglia in plaque samples compared to non-plaque controls whereas only 1 gene, C $4 b$, of genes associated with peripheral macrophages displayed significant expression in plaque compared to non-plaque samples. For whole cortex samples, half of the top 25 microglial genes were significantly increased in plaque samples compared to non-plaque. (JPG $43 \mathrm{~kb}$ )

\section{Abbreviations}

AD: Alzheimer's disease; APP: Amyloid precursor protein; Aß: Amyloid-beta; CNS: Central nervous system; DAM: Disease-associated microglia; GWAS: Genome-wide association studies; ITAM: Immunoreceptor tyrosinebased activation motif; ITIM: Immunoreceptor tyrosine-based inhibition motif; LOAD: Late-onset Alzheimer's disease

\section{Availability of data and materials}

The datasets used and/or analyzed during the current study are available from the corresponding author upon acceptance.

\section{Authors' contributions \\ SMR contributed to study design, performed the experiments, and contributed to writing of the manuscript. KQT contributed to study design, performed statistical analysis, and contributed to writing of the manuscript. PG and JM aided with project execution and edited the manuscript. VM and JG carried out bioinformatics and analysis. MP, RS, and VM prepared samples. CW provided in situ histological samples, imaging, and analysis. EOL contributed to writing of the manuscript, figure construction, and carried analysis. GZ contributed to histological data. RS prepared the samples for LCM experiments and data analysis. BJH, JK, and MK all critically reviewed and edited the manuscript. CM supervised the project and contributed to the study design, experiments, analysis, and writing of the manuscript. All authors read and approved the final manuscript.}

\section{Ethics approval and consent to participate}

Principles of laboratory animal care were followed, and all studies were previously approved by the Institutional Animal Care and Use Committee and were performed in accordance to the Guide for the Care and Use of Laboratory Animals as adopted and promulgated by the National Institutes of Health (Library of Congress Control Number 2010940400, revised 2011).

\section{Consent for publication}

Not applicable

\section{Competing interests}

All authors were or are employees and shareholders in Merck \& Co., Inc. at the time of their contributions. The authors declare that they have no competing interests.

\section{Publisher's Note}

Springer Nature remains neutral with regard to jurisdictional claims in published maps and institutional affiliations.

\section{Author details}

${ }^{1}$ In Vivo Pharmacology, Merck \& Co, Kenilworth, New Jersey, USA. ${ }^{2}$ Genetics and Genomics, Merck \& Co., West Point, Pennsylvania, USA. ${ }^{3}$ Neuroscience, Merck \& Co., Merck Research Labs, Boston, Massachusetts, USA. ${ }^{4}$ Genetics and Genomics, Merck \& Co., Merck Research Labs, Boston, Massachusetts, USA. ${ }^{5}$ Neuroscience, Merck \& Co, West Point, Pennsylvania, USA. ${ }^{6}$ Systems Toxicology, Merck \& Co., Kenilworth, New Jersey, USA. ${ }^{7}$ Infectious Diseases and Vaccines, Merck \& Co., West Point, Kenilworth, Pennsylvania, USA.

Received: 2 February 2018 Accepted: 1 August 2018

Published online: 06 September 2018

\section{References}

1. Duyckaerts C, Delatour B, Potier MC. Classification and basic pathology of Alzheimer disease. Acta Neuropathol. 2009;118:5-36.

2. Guillot-Sestier MV, Doty KR, Town T. Innate immunity fights Alzheimer's disease. Trends Neurosci. 2015:38:674-81.

3. Yan R, Vassar R. Targeting the beta secretase BACE1 for Alzheimer's disease therapy. Lancet Neurol. 2014;13:319-29.

4. Orre M, Kamphuis W, Osborn LM, Jansen AHP, Kooijman L, Bossers K, Hol EM. Isolation of glia from Alzheimer's mice reveals inflammation and dysfunction. Neurobiol Aging. 2014;35:2746-60.

5. Gjoneska E, Pfenning AR, Mathys H, Quon G, Kundaje A, Tsai LH, Kellis M. Conserved epigenomic signals in mice and humans reveal immune basis of Alzheimer's disease. Nature. 2015;518:365-9.

6. Nimmerjahn A, Kirchhoff F, Helmchen F. Resting microglial cells are highly dynamic surveillants of brain parenchyma in vivo. Science. 2005:308:1314-8.

7. Ager RR, Fonseca MI, Chu SH, Sanderson SD, Taylor SM, Woodruff TM, Tenner AJ. Microglial C5aR (CD88) expression correlates with amyloid-beta deposition in murine models of Alzheimer's disease. J Neurochem. 2010; 113:389-401.

8. Arends YM, Duyckaerts C, Rozemuller JM, Eikelenboom P, Hauw JJ. Microglia, amyloid and dementia in alzheimer disease. A correlative study. Neurobiol Aging. 2000;21:39-47.

9. Rodriguez GA, Tai LM, LaDu MJ, Rebeck GW. Human APOE4 increases microglia reactivity at Abeta plaques in a mouse model of Abeta deposition. J Neuroinflammation. 2014;11:111

10. Aisen PS, Schafer KA, Grundman M, Pfeiffer E, Sano M, Davis KL, Farlow MR, Jin S, Thomas RG, Thal LJ. Alzheimer's disease cooperative S: effects of rofecoxib or naproxen vs placebo on Alzheimer disease progression: a randomized controlled trial. JAMA. 2003;289:2819-26.

11. Aisen PS, Schmeidler J, Pasinetti GM. Randomized pilot study of nimesulide treatment in Alzheimer's disease. Neurology. 2002;58:1050-4.

12. Green RC, Schneider LS, Amato DA, Beelen AP, Wilcock G, Swabb EA, Zavitz $\mathrm{KH}$. Tarenflurbil phase 3 study G: effect of tarenflurbil on cognitive decline and activities of daily living in patients with mild Alzheimer disease: a randomized controlled trial. JAMA. 2009:302:2557-64.

13. Pasqualetti $P$, Bonomini C, Dal Forno G, Paulon L, Sinforiani E, Marra C, Zanetti $\mathrm{O}$, Rossini PM. A randomized controlled study on effects of ibuprofen on cognitive progression of Alzheimer's disease. Aging Clin Exp Res. 2009;21:102-10.

14. Reines SA, Block GA, Morris JC, Liu G, Nessly ML, Lines CR, Norman BA, Baranak CC. Rofecoxib protocol 091 study G: Rofecoxib: no effect on Alzheimer's disease in a 1-year, randomized, blinded, controlled study. Neurology. 2004;62:66-71.

15. Bettens K, Sleegers K, Van Broeckhoven C. Genetic insights in Alzheimer's disease. Lancet Neurol. 2013;12:92-104. 
16. Bradshaw EM, Chibnik LB, Keenan BT, Ottoboni L, Raj T, Tang A, Rosenkrantz LL, Imboywa S, Lee M, Von Korff A, et al. CD33 Alzheimer's disease locus: altered monocyte function and amyloid biology. Nat Neurosci. 2013;16:848-50

17. Hollingworth P, Harold D, Sims R, Gerrish A, Lambert JC, Carrasquillo MM, Abraham R, Hamshere ML, Pahwa JS, Moskvina V, et al. Common variants at ABCA7, MS4A6A/MS4A4E, EPHA1, CD33 and CD2AP are associated with Alzheimer's disease. Nat Genet. 2011;43:429-35.

18. Jonsson T, Stefansson H, Steinberg S, Jonsdottir I, Jonsson PV, Snaedal J, Bjornsson S, Huttenlocher J, Levey Al, Lah JJ, et al. Variant of TREM2 associated with the risk of Alzheimer's disease. N Engl J Med. 2013;368:107-16.

19. Lambert JC, Heath S, Even G, Campion D, Sleegers K, Hiltunen M, Combarros O, Zelenika D, Bullido MJ, Tavernier B, et al. Genome-wide association study identifies variants at CLU and CR1 associated with Alzheimer's disease. Nat Genet. 2009:41:1094-9.

20. Lambert JC, Ibrahim-Verbaas CA, Harold D, Naj AC, Sims R, Bellenguez C, DeStafano AL, Bis JC, Beecham GW, Grenier-Boley B, et al. Meta-analysis of 74,046 individuals identifies 11 new susceptibility loci for Alzheimer's disease. Nat Genet. 2013:45:1452-8.

21. Seshadri S, Fitzpatrick AL, Ikram MA, DeStefano AL, Gudnason V, Boada M, Bis JC, Smith AV, Carassquillo MM, Lambert JC, et al. Genome-wide analysis of genetic loci associated with Alzheimer disease. JAMA. 2010;303:1832-40.

22. Villegas-Llerena C, Phillips A, Garcia-Reitboeck P, Hardy J, Pocock JM. Microglial genes regulating neuroinflammation in the progression of Alzheimer's disease. Curr Opin Neurobiol. 2016;36:74-81.

23. Zhang B, Gaiteri C, Bodea LG, Wang Z, McElwee J, Podtelezhnikov AA, Zhang C, Xie T, Tran L, Dobrin R, et al. Integrated systems approach identifies genetic nodes and networks in late-onset Alzheimer's disease. Cell. 2013;153:707-20.

24. Efthymiou AG, Goate AM. Late onset Alzheimer's disease genetics implicates microglial pathways in disease risk. Mol Neurodegener. 2017;12:43.

25. Bianchin MM, Capella HM, Chaves DL, Steindel M, Grisard EC, Ganev GG, da Silva Junior JP, Neto Evaldo S, Poffo MA, Walz R, et al. Nasu-Hakola disease (polycystic lipomembranous osteodysplasia with sclerosing leukoencephalopathy--PLOSL): a dementia associated with bone cystic lesions. From clinical to genetic and molecular aspects. Cell Mol Neurobiol. 2004:24:1-24.

26. Chouery E, Delague V, Bergougnoux A, Koussa S, Serre JL, Megarbane A. Mutations in TREM2 lead to pure early-onset dementia without bone cysts. Hum Mutat. 2008:29:E194-204.

27. Giraldo M, Lopera F, Siniard AL, Corneveaux JJ, Schrauwen I, Carvajal J, Munoz C, Ramirez-Restrepo M, Gaiteri C, Myers AJ, et al. Variants in triggering receptor expressed on myeloid cells 2 are associated with both behavioral variant frontotemporal lobar degeneration and Alzheimer's disease. Neurobiol Aging. 2013;2077(34):e2011-78.

28. Montalbetti L, Ratti MT, Greco B, Aprile C, Moglia A, Soragna D. Neuropsychological tests and functional nuclear neuroimaging provide evidence of subclinical impairment in Nasu-Hakola disease heterozygotes. Funct Neurol. 2005;20:71-5.

29. Guerreiro R, Wojtas A, Bras J, Carrasquillo M, Rogaeva E, Majounie E, Cruchaga C, Sassi C, Kauwe JS, Younkin S, et al. TREM2 variants in Alzheimer's disease. N Engl J Med. 2013;368:117-27.

30. Condello C, Yuan P, Schain A, Grutzendler J. Microglia constitute a barrier that prevents neurotoxic protofibrillar Abeta42 hotspots around plaques. Nat Commun. 2015;6:6176.

31. Frank S, Burbach GJ, Bonin M, Walter M, Streit W, Bechmann I, Deller T TREM2 is upregulated in amyloid plaque-associated microglia in aged APP23 transgenic mice. Glia. 2008:56:1438-47.

32. Griciuc A, Serrano-Pozo A, Parrado AR, Lesinski AN, Asselin CN, Mullin K, Hooli B, Choi SH, Hyman BT, Tanzi RE. Alzheimer's disease risk gene CD33 inhibits microglial uptake of amyloid beta. Neuron. 2013;78:631-43.

33. Hsiao K, Chapman P, Nilsen S, Eckman C, Harigaya Y, Younkin S, Yang F, Cole G. Correlative memory deficits, Abeta elevation, and amyloid plaques in transgenic mice. Science. 1996;274:99-102.

34. Podtelezhnikov AA, Tanis KQ, Nebozhyn M, Ray WJ, Stone DJ, Loboda AP. Molecular insights into the pathogenesis of Alzheimer's disease and its relationship to normal aging. PLoS One. 2011;6:e29610.

35. Liao L, Cheng D, Wang J, Duong DM, Losik TG, Gearing M, Rees HD, Lah JJ, Levey Al, Peng J. Proteomic characterization of postmortem amyloid plaques isolated by laser capture microdissection. J Biol Chem. 2004; 279:37061-8.
36. Hickman SE, Kingery ND, Ohsumi TK, Borowsky ML, Wang LC, Means TK, E Khoury J. The microglial sensome revealed by direct RNA sequencing. Nat Neurosci. 2013;16:1896-905.

37. Keren-Shaul H, Spinrad A, Weiner A, Matcovitch-Natan O, Dvir-Szternfeld R, Ulland TK, David E, Baruch K, Lara-Astaiso D, Toth B, et al. A unique microglia type associated with restricting development of Alzheimer's disease. Cell. 2017;169:1276-90. e1217

38. Aggarwal NR, King LS, D'Alessio FR. Diverse macrophage populations mediate acute lung inflammation and resolution. Am J Physiol Lung Cell Mol Physiol. 2014;306:L709-25.

39. Butovsky O, Jedrychowski MP, Moore CS, Cialic R, Lanser AJ, Gabriely G, Koeglsperger T, Dake B, Wu PM, Doykan CE, et al. Identification of a unique TGF-beta-dependent molecular and functional signature in microglia. Nat Neurosci. 2014;17:131-43.

40. Heneka MT, Carson MJ, El Khoury J, Landreth GE, Brosseron F, Feinstein DL, Jacobs AH, Wyss-Coray T, Vitorica J, Ransohoff RM, et al. Neuroinflammation in Alzheimer's disease. Lancet Neurol. 2015:14:388-405.

41. Mullan M, Crawford F, Axelman K, Houlden H, Lilius L, Winblad B, Lannfelt L. A pathogenic mutation for probable Alzheimer's disease in the APP gene at the N-terminus of beta-amyloid. Nat Genet. 1992;1:345-7.

42. Bornemann KD, Staufenbiel M. Transgenic mouse models of Alzheimer's disease. Ann N Y Acad Sci. 2000;908:260-6.

43. Sasaguri H, Nilsson P, Hashimoto S, Nagata K, Saito T, De Strooper B, Hardy J, Vassar R, Winblad B, Saido TC. APP mouse models for Alzheimer's disease preclinical studies. EMBO J. 2017;36:2473-87.

44. Gu L, Guo Z. Alzheimer's Abeta42 and Abeta40 peptides form interlaced amyloid fibrils. J Neurochem. 2013;126:305-11.

45. Jan A, Gokce O, Luthi-Carter R, Lashuel HA. The ratio of monomeric to aggregated forms of Abeta 40 and Abeta42 is an important determinant of amyloid-beta aggregation, fibrillogenesis, and toxicity. J Biol Chem. 2008; 283:28176-89.

46. Kuperstein I, Broersen K, Benilova I, Rozenski J, Jonckheere W, Debulpaep M, Vandersteen A, Segers-Nolten I, Van Der Werf K, Subramaniam V, et al. Neurotoxicity of Alzheimer's disease Abeta peptides is induced by small changes in the Abeta42 to Abeta40 ratio. EMBO J. 2010;29:3408-20.

47. Pauwels K, Williams TL, Morris KL, Jonckheere W, Vandersteen A, Kelly G, Schymkowitz J, Rousseau F, Pastore A, Serpell LC, Broersen K. Structural basis for increased toxicity of pathological abeta42:abeta40 ratios in Alzheimer disease. J Biol Chem. 2012;287:5650-60.

48. Snyder SW, Ladror US, Wade WS, Wang GT, Barrett LW, Matayoshi ED, Huffaker HJ, Krafft GA, Holzman TF. Amyloid-beta aggregation: selective inhibition of aggregation in mixtures of amyloid with different chain lengths. Biophys J. 1994;67:1216-28.

49. Yoshiike Y, Chui DH, Akagi T, Tanaka N, Takashima A. Specific compositions of amyloid-beta peptides as the determinant of toxic beta-aggregation. J Biol Chem. 2003;278:23648-55.

50. McGowan E, Pickford F, Kim J, Onstead L, Eriksen J, Yu C, Skipper L, Murphy MP, Beard J, Das P, et al. Abeta42 is essential for parenchymal and vascular amyloid deposition in mice. Neuron. 2005;47:191-9.

51. Frautschy SA, Yang F, Irrizarry M, Hyman B, Saido TC, Hsiao K, Cole GM. Microglial response to amyloid plaques in APPsw transgenic mice. Am J Pathol. 1998;152:307-17.

52. Gyoneva S, Swanger SA, Zhang J, Weinshenker D, Traynelis SF. Altered motility of plaque-associated microglia in a model of Alzheimer's disease. Neuroscience. 2016;330:410-20.

53. Itagaki S, McGeer PL, Akiyama H, Zhu S, Selkoe D. Relationship of microglia and astrocytes to amyloid deposits of Alzheimer disease. J Neuroimmunol. 1989;24:173-82.

54. Perlmutter LS, Barron E, Chui HC. Morphologic association between microglia and senile plaque amyloid in Alzheimer's disease. Neurosci Lett. 1990;119:32-6.

55. Simard AR, Soulet D, Gowing G, Julien JP, Rivest S. Bone marrow-derived microglia play a critical role in restricting senile plaque formation in Alzheimer's disease. Neuron. 2006:49:489-502.

56. Stalder M, Phinney A, Probst A, Sommer B, Staufenbiel M, Jucker M. Association of microglia with amyloid plaques in brains of APP23 transgenic mice. Am J Pathol. 1999;154:1673-84.

57. Yin Z, Raj D, Saiepour N, Van Dam D, Brouwer N, Holtman IR, Eggen BJL, Moller T, Tamm JA, Abdourahman A, et al. Immune hyperreactivity of Abeta plaque-associated microglia in Alzheimer's disease. Neurobiol Aging. 2017; 55:115-22. 
58. Geissmann F, Gordon S, Hume DA, Mowat AM, Randolph GJ. Unravelling mononuclear phagocyte heterogeneity. Nat Rev Immunol. 2010;10:453-60.

59. Prinz M, Priller J. Microglia and brain macrophages in the molecular age: from origin to neuropsychiatric disease. Nat Rev Neurosci. 2014;15:300-12.

60. Farfel JM, Yu L, Buchman AS, Schneider JA, De Jager PL, Bennett DA. Relation of genomic variants for Alzheimer disease dementia to common neuropathologies. Neurology. 2016;87:489-96.

61. Schott JM, Crutch SJ, Carrasquillo MM, Uphill J, Shakespeare TJ, Ryan NS, Yong KX, Lehmann M, Ertekin-Taner N, Graff-Radford NR, et al. Genetic risk factors for the posterior cortical atrophy variant of Alzheimer's disease. Alzheimers Dement. 2016;12:862-71.

62. Chouraki V, Reitz C, Maury F, Bis JC, Bellenguez C, Yu L, Jakobsdottir J Mukherjee S, Adams HH, Choi SH, et al. Evaluation of a genetic risk score to improve risk prediction for Alzheimer's disease. J Alzheimers Dis. 2016;53:921-32

63. Painter MM, Atagi Y, Liu CC, Rademakers R, Xu H, Fryer JD, Bu G. TREM2 in CNS homeostasis and neurodegenerative disease. Mol Neurodegener. 2015;10:43.

64. Takahashi K, Rochford CD, Neumann H. Clearance of apoptotic neurons without inflammation by microglial triggering receptor expressed on myeloid cells-2. J Exp Med. 2005;201:647-57.

65. Atagi Y, Liu CC, Painter MM, Chen XF, Verbeeck C, Zheng H, Li X, Rademakers R, Kang SS, Xu H, et al. Apolipoprotein E is a ligand for triggering receptor expressed on myeloid cells 2 (TREM2). J Biol Chem. 2015;290:26043-50.

66. Bailey CC, DeVaux LB, Farzan M. The triggering receptor expressed on myeloid cells 2 binds apolipoprotein E. J Biol Chem. 2015;290:26033-42.

67. Wang Y, Cella M, Mallinson K, Ulrich JD, Young KL, Robinette ML, Gilfillan S, Krishnan GM, Sudhakar S, Zinselmeyer BH, et al. TREM2 lipid sensing sustains the microglial response in an Alzheimer's disease model. Cell. 2015; 160:1061-71.

68. Yeh FL, Wang Y, Tom I, Gonzalez LC, Sheng M. TREM2 binds to apolipoproteins, including APOE and CLU/APOJ, and thereby facilitates uptake of amyloid-beta by microglia. Neuron. 2016;91:328-40.

69. Kleinberger $G$, Yamanishi $Y$, Suarez-Calvet M, Czirr E, Lohmann E, Cuyvers E, Struyfs H, Pettkus N, Wenninger-Weinzierl A, Mazaheri F, et al. TREM2 mutations implicated in neurodegeneration impair cell surface transport and phagocytosis. Sci Transl Med. 2014;6:243ra286.

Ready to submit your research? Choose BMC and benefit from:

- fast, convenient online submission

- thorough peer review by experienced researchers in your field

- rapid publication on acceptance

- support for research data, including large and complex data types

- gold Open Access which fosters wider collaboration and increased citations

- maximum visibility for your research: over $100 \mathrm{M}$ website views per year

At $\mathrm{BMC}$, research is always in progress.

Learn more biomedcentral.com/submissions 